\title{
Characterization of Enterococcus faecalis isolates by chicken embryo lethality assay and ERIC-PCR
}

\author{
Ana E. Blanco, Martin Barz, David Cavero, Wiebke Icken, A. Reza Sharific, Matthias Voss, Carlos \\ Buxadé and Rudolf Preisinger
}

\begin{abstract}
Enterococcus faecalis is the major causative agent of amyloid arthropathy in chickens. Given the difficulty of estimating the risk from field strains, the embryo lethality assay (ELA) is proposed in this study as a model to predict the virulence of 68 avian E. faecalis strains. Additionally, Enterobacterial Repetitive Intergenic Consensus Polymerase Chain Reaction (ERIC-PCR) was used to characterize the genetic diversity of the $E$. faecalis strains. The ELA was performed 10 times with subsets of 7-8 E. faecalis strains each on a sample of 9987 eggs, including control groups. An estimated 3-24 colony-forming units were inoculated into the allantoic cavity of 10-day-old embryos. The embryonic mortality rate (EMR) was determined by means of candling the eggs over a period of seven days. The ELA was able to distinguish the virulence of the E. faecalis strains. Twenty-six strains were considered as avirulent strains with an EMR of below $40 \%$. Five strains were highly virulent with an EMR above $80 \%$. The remaining 37 strains were classified as strains of moderate virulence, causing an EMR between $40 \%$ and $80 \%$. The highest EMR occurred three and four days post-inoculation (p.i.). From the fourth day p.i., almost no embryonic mortality was observed. Therefore, the ELA could be optimized by reducing experiment duration to four days p.i. ERIC-PCR did not cluster the strains according to its virulence, although ERIC banding patterns revealed a considerable genetic diversity. In conclusion, the ELA can be considered a reliable and useful tool to predict the virulence of avian $E$. faecalis strains.
\end{abstract}

\section{KEYWORDS \\ Chicken; Enterococcus faecalis; embryo lethality assay; ERIC-PCR; amyloid arthropathy}

\section{Introduction}

Amyloid arthropathy (AA) in chickens is characterized by AA amyloid depositions in the knee and hock joints, lameness and growth depression, which compromises the welfare of chickens and has a significant economic impact since the disease affects about $20-30 \%$ of all European chicken flocks (Landman et al., 1998). AA is the only amyloidosis type that has been associated with chronic infections induced by arthropathic and amyloidogenic Enterococcus faecalis (E. faecalis) strains so far (Landman et al., 1997).

Treatment of AA has focused to date on the prevention and treatment of chronic inflammation produced by $E$. faecalis strains, as recently reviewed by Blanco et al. (2016). However, the enterococcal infection treatments are increasingly limited. This, on the one hand, is due to the restrictions on antimicrobial use in animal production emerged since the global health crisis of antimicrobial resistance (World Health Organization, 2012). On the other hand, it is due to the zoonotic potential of the E. faecalis strains, its ability to acquire antimicrobial resistance and its capacity to acquire virulence genes by transfer of pathogenicity islands (Coburn et al., 2007).
The molecular typing methods have identified several Sequence types (STs) within the E. faecalis population associated with different diseases. Petersen et al. (2009) observed through Multilocus Sequence Typing (MLST) the specific association between AA and the Sequence Type (ST) 82 of E. faecalis, which has been related with this disease worldwide (Petersen et al., 2007). Additionally, according to the data base from the Multi Locus Sequence Typing website (http://www.mlst.net), ST36, ST59, ST82, ST170, ST171, ST172 and ST174 of E. faecalis have been also associated with AA, and ST34, ST82, ST174 and ST177 have been associated with first week mortality in layers (Olsen et al., 2012b). In broilers, ST32, ST176, ST177 and ST249 have been related with AA, and ST16, ST82, ST174 and ST249 have the capacity of inducing septicaemia and/or salpingitis. Of all these STs, ST82, ST174 and ST177 constitute more than $80 \%$ of the mortality related with broiler breeders (Gregersen et al., 2010). It should be noted that the ST36, ST59 and ST82 identified in avian E. faecalis strains have also been associated to human infections which may represent a zoonosis (http://www.mlst.net, Ruiz-Garbajosa et al., 2006). 
The characterization of E. faecalis allows the identification of possible virulence genes: aggregation substance (asal or agg), endocarditis antigen (efaA), cytolisine (cylA), gelatinase ( $g e l E)$, collagen-binding protein (ace), hyaluronidase (hyl) and extracellular surface proteins (esp). Ciftci \& Diker (2009) reported that the strains with absence of $g e l E$ expression inoculated in chickens did not develop any pathological signs of AA, whereas $65.2 \%$ of gelE positive strains caused AA. However, the virulence genes cannot be the only cause of virulence of E. faecalis strains in poultry since these virulence genes have been found in both commensal and pathogenic avian E. faecalis isolates (Olsen et al., 2012a).

Additionally, E. faecalis possesses a natural intrinsic resistance towards several antimicrobials (Hayes et al., 2003) such as chloramphenicol, clindamycin, erythromycin, tetracycline, high-level aminoglycosides, betalactamase and vancomycin (McBride et al., 2007). Consequently, this bacterium is able to survive in an environment in which antimicrobial agents are heavily used and, therefore, its treatment becomes more complicated. A thorough understanding of the risk from field E. faecalis strains is therefore needed in order to develop effective control strategies against bacterial infections to reduce the incidence rate of AA and, consequently, to guarantee the welfare of the birds and improve their economic performance.

The embryo lethality assay (ELA) has previously demonstrated its ability to discriminate between virulent and avirulent isolates of fungi (Jacobsen et al., 2010), and bacteria such as Escherichia coli (Nolan et al., 1992; Wooley et al., 2000; Gibbs et al., 2003; Gibbs \& Wooley, 2003; Montgomery et al., 2005; Oh et al., 2012), Yersinia enterocolitica (Townsend et al., 2008), Campylobacter jejuni (Stewart-Tull et al., 2009), Staphylococcus aureus (Polakowska et al., 2012), Riemerella anatipestifer (Seo et al., 2013) and Listeria monocytogenes (Andersson et al., 2015). In the case of Enterococcus spp., Borst et al. (2014) reported that the ELA is able to differentiate between virulent and avirulent E. cecorum strains, whose genotype was closely related each other through pulsedfield gel electrophoresis. Rudolph (2004) observed that the E. faecalis strains produced different degrees of mortality when the embryos were infected in the allantoic cavity (AC) with an infectious dose of 2500 colonyforming units $(\mathrm{CFU}) / \mathrm{ml}$, and Blanco et al. (2017) reported on the importance of establishing an appropriate infectious dose of $E$. faecalis in order to avoid a massive embryonic mortality that would not allow discrimination among the E. faecalis strains. These authors optimized and standardized the ELA to evaluate the virulence of the avian E. faecalis strain K923/96 and reported its median lethality dose $\left(\mathrm{LD}_{50}\right)$ in chicken embryos.

Therefore, the main goal of this study was to characterize different avian E. faecalis strains through the
ELA taking the E. faecalis strain K923/96 and the infectious dose of $5 \mathrm{CFU} / \mathrm{ml}$ reported previously by Blanco et al. (2017) as reference, and thus corroborate the ELA capacity for discriminating E. faecalis strains. It was of particular interest to characterize the genetic diversity of these strains by Enterobacterial Repetitive Intergenic Consensus (ERIC) Polymerase Chain Reaction (PCR) under the hypothesis that strains with similar DNA banding patterns may be similar also in their infective ability.

\section{Materials and methods}

\section{Ethical statement}

Avian embryos are currently not regulated by legislation as animal experiments in Germany (https:// www.gesetze-im-internet.de/tierschg/BJNR012770972. $\mathrm{html}$ ) and, therefore, an approval from an ethics committee was not required for this study. In addition, the ELAs in chicken embryos were completed four days prior to hatching, on developmental day 17 at the latest.

\section{Bacterial strains}

A total of 69 avian strains of Enterococcus spp. (1 E. hirae and 68 E. faecalis strains) were characterized in the present study through 10 ELAs (I to X). As shown in Figure 1, most of the known E. faecalis strains were obtained from clinical cases of AA at layer farms in Germany $(n=31)$, France $(n=2)$ and Switzerland $(n=1)$. Only three E. faecalis strains (282-4, 513-5 and 515-3) were isolated from apparently healthy birds. Unfortunately, the information on the isolation from 34 strains is unknown. All strains were obtained during 2011 and 2012, except the E. faecalis strain K923/96, which was isolated in 1996 and it has been well-characterized and used in different studies by Rudolph (2004), Petersen et al. (2009) and Blanco et al. (2017), and the strains 1082-01 and 607-01 isolated in 2004 and characterized by Petersen et al. (2009). Additionally, 49 E. faecalis strains used in the present study were tested before by the laboratory of Lohmann Tierzucht GmbH (Germany) as ST82 by using the Real Time (RT-) PCR method described by Petersen et al. (2010). The genes ace, efaA, cyl, asal (or agg) and gelE were present in all these strains although the genes esp and $h y l$ were not determined.

\section{Enterobacterial Repetitive Intergenic Consensus (ERIC) Polymerase Chain Reaction (PCR) and phylogenetic analysis}

All Enterococcus spp., except the E. faecalis strain 232396, were used to develop the ERIC-PCR method 


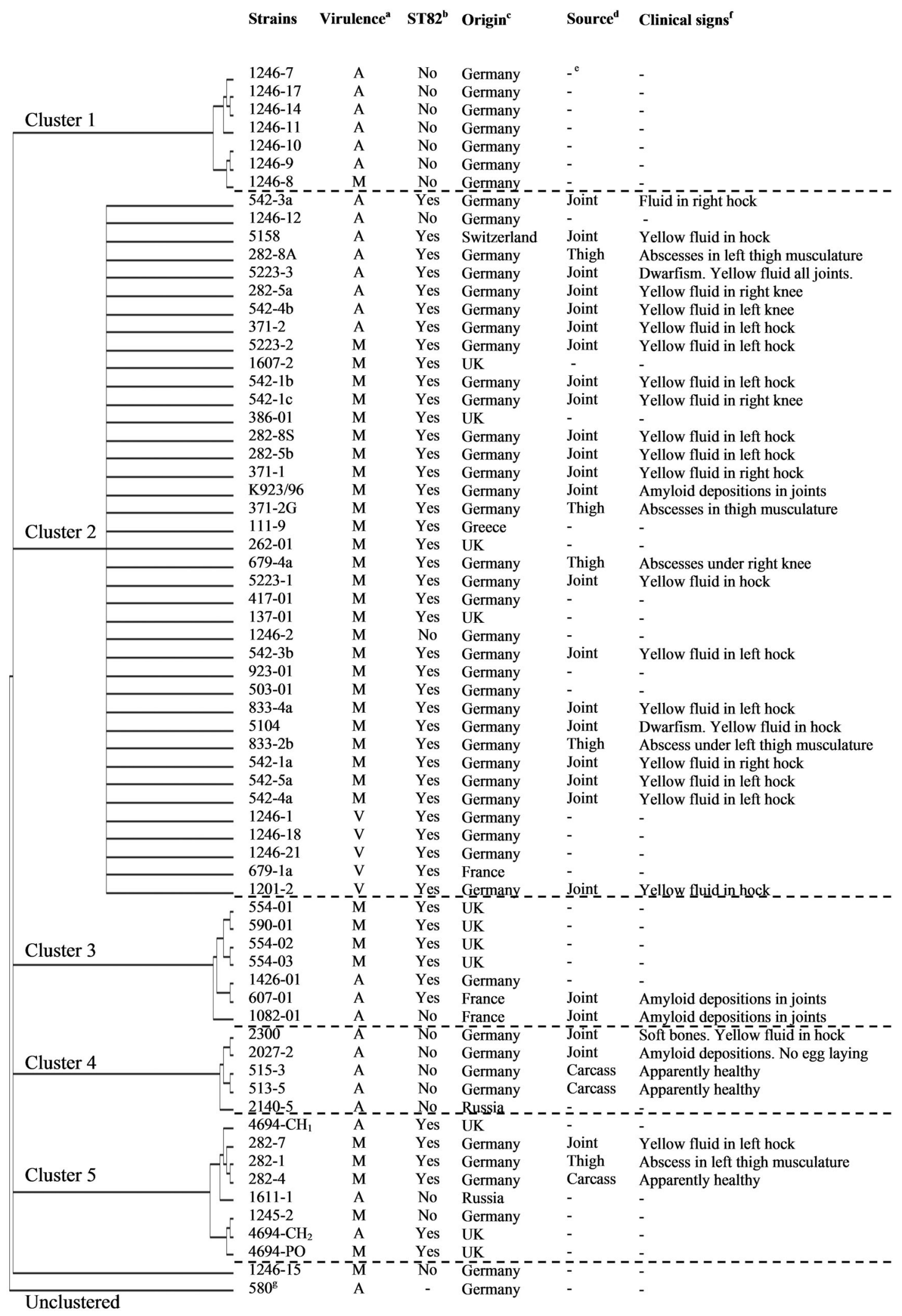

Figure 1. Phylogenetic tree (drawn in the rectangular cladogram format) of one E. hirae and $67 \mathrm{E}$. faecalis strains based on the aligned nucleotide sequences after ERIC-PCR. ${ }^{\text {a }}$ Virulence classification of the strains through the ELAs ( $A=A$ virulent, $M=$ Moderate and $\mathrm{V}=$ Virulent). ${ }^{\mathrm{b}}$ ST 82 refers to the sequence type 82 reported by the laboratory of Lohmann Tierzucht GmbH (Germany) by using the RT-PCR. ${ }^{c}$ Country of origin of the strain. ${ }^{d}$ Source of isolation. ${ }^{e}$ This information is unknown. ${ }^{f}$ Clinical signs observed in the birds. ${ }^{9}$ E. hirae strain used as an outgroup.

described by Jurkovič et al. (2007). The bacteria, which were preserved in a CRYOBANK ${ }^{\mathrm{TM}}$ tube, were recovered removing the cryobank tube from the freezer.
Bacteria were cultured on a sheep blood agar plate. The agar plate was incubated aerobically for $24 \mathrm{~h}$ at $37^{\circ} \mathrm{C}$. After incubation, colonies were scraped off and 
a suspension of $400 \mu$ l was prepared in phosphate-buffered saline (PBS). DNA was purified using the NucleoSpin Tissue Kit (MachereyNagel, Düren, Germany). Afterwards $3 \mu \mathrm{l}$ of the suspension was used for PCR amplification. Isolates were genotyped by ERIC-PCR. Reactions were carried out in a total volume of $50 \mu \mathrm{l}$ containing $18 \mu \mathrm{l}$ of RNAse free water, $3 \mu \mathrm{l}$ of $\mathrm{MgCl}_{2}, 25 \mu \mathrm{l}$ of dNTPs mix $(10 \mathrm{mM}$ of each), $1 \mu \mathrm{l}$ of ERIC1-R primer (5'-ATGTAAGCTCC TGGGGATTCAC-3) and $3 \mu \mathrm{l}$ of template DNA. The ERIC-PCR was carried out with only one single ERIC 1 primer, which uses the total DNA and, therefore, provides results with good reproducibility. Furthermore, its discriminatory power is higher than when using both ERIC 1 and ERIC 2 primers (Jurkovič et al., 2007). Amplifications were performed in an Eppendorf thermocycler with a cycling program consisting of an initial denaturing step at $94^{\circ} \mathrm{C}$ for $5 \mathrm{~min}$, then 35 cycles of denaturation at $94^{\circ} \mathrm{C}$ for $1 \mathrm{~min}$, annealing at $48^{\circ} \mathrm{C}$ for $1 \mathrm{~min}$ and elongation at $72^{\circ} \mathrm{C}$ for $2 \mathrm{~min}$ and a final extension of $72^{\circ} \mathrm{C}$ for $7 \mathrm{~min}$.

ERIC-PCR results show banding patterns, which can easily be visualized by agarose gel electrophoresis. The genetic diversity of the chromosomal DNA may be explained by differences in the banding patterns among the strains (Odinot et al., 1995). Therefore, all products were analysed by electrophoresis through $1.5 \%$ agarose gels at $70 \mathrm{~V}$ for $70 \mathrm{~min}$ in a $1 \mathrm{x}$ TAE buffer (40 mM Tris-acetate, $\mathrm{pH} 8.3$ ) and revelled in ethidium bromide $(10 \mathrm{mg} / \mathrm{ml})$. The gels were visualized and photographed under UV transilluminator.

The phylogenetic analysis was performed based on the banding pattern of the different strains of Enterococcus spp. by using the Phylogenetic Inference Package, PHYLIP-3.68 (Felsenstein, 2005), after ERICPCR. The data were calculated by using parsimony. The tree was visualized using the program Archaeopteryx 0.9901 beta (Han \& Zmasek, 2009).

\section{Embryo lethality assay}

The ELAs were performed on a sample of 9987 eggs of White Layers at an age of 29-62 weeks. The tested flocks were free from diseases such as Mycoplasma or Salmonella. Only first-quality hatching eggs were used - i.e. soiled eggs as well as eggs with hairline cracks were sorted out. Fertilized eggs were stored at $15^{\circ} \mathrm{C}$ for a maximum of four days prior to incubation and incubated at $37.8^{\circ} \mathrm{C}$ and humidity from $52 \%$ to $56 \%$ with an automatic turning.

Each individual ELA was composed of a subset of eight strains, with the exception of the ELA IX, in which were used seven strains. The known and wellcharacterized pathogenic avian E. faecalis strain K923/96 (Rudolph, 2004; Petersen et al., 2009; Blanco et al., 2017) was included in all ELAs as the positive control (reference strain). Fertilized eggs were inoculated via the $\mathrm{AC}$ after 10 days of incubation. In addition to the infected embryos, there was always one negative control group in each ELA, which was inoculated in the AC with $0.2 \mathrm{ml}$ of sterile PBS. In each ELA, 100 eggs per strain and control groups were used, with the exception of two negative control groups (ELA I and II), in which 177 and 110 eggs were used, respectively.

The inoculum was prepared as previously described by Blanco et al. (2017) up to an infectious dose of $5 \mathrm{CFU} / \mathrm{ml}$, which corresponds approximately with the $\mathrm{LD}_{50}$ of the reference strain K923/96. By an inoculation volume of $0.2 \mathrm{ml} / \mathrm{egg}$, the number of bacteria used for inoculation was one CFU/egg. However, the number of counted colonies may vary between 500 and $5000 \mathrm{CFU} / \mathrm{ml}$ (Rudolph, 2004). Therefore, the number of CFU contained in each dose was verified by viable count on a sheep blood agar plate in order to guarantee that the infectious dose was correctly prepared. The number of bacteria inoculated into the embryos ranged from 15 to $120 \mathrm{CFU} / \mathrm{ml}$ (i.e. from 3 to $24 \mathrm{CFU} / \mathrm{egg}$ ), depending on the strain. In the case of the E. hirae strain, the embryos were inoculated with $275 \mathrm{CFU} / \mathrm{ml}$ (55 CFU/egg). The real number of CFU of each strain inoculated into the embryos is shown in Figure 2.

The incubated eggs were candled every $24 \mathrm{~h}$ for seven days after infection, and embryonic mortalities were daily recorded per strain and control groups. The number of dead embryos was used to classify the virulence of the E. faecalis strains. As previously reported by Rudolph (2004), the strains with an embryonic mortality rate (EMR) below $40 \%$ were considered avirulent strains; above $80 \%$ virulent strains and between $40 \%$ and $80 \%$ were classified as strains of moderate virulence. It should be noted that the embryos that died within $24 \mathrm{~h}$ p.i. were removed from the experiment, since their deaths could be attributed to a lethal trauma during the manipulation (Wooley et al., 2000).

At the end of each ELA, at 17 days of incubation, all surviving embryos were sacrificed by hypothermia. They were stored for $48 \mathrm{~h}$ at $4^{\circ} \mathrm{C}$.

Random selections of the embryos killed by the infection, as well as some surviving embryos at the end of the ELAs, were used to evaluate macroscopic lesions and to re-isolate the strains from the allantoic fluid of the embryos, as reported by Blanco et al. (2017).

\section{Statistical analysis}

Statistical analyses of mortality data were carried out by applying a linear logistic mixed model with a binary response variable, which was modelled as a binomial random variable $\left(y_{i}\right)$. The dependent variable $\left(y_{i}\right)$ can take the value 1 with a probability for embryonic mortality $\pi_{i}$ or the value 0 with a probability to survive of 1 $-\pi_{i}$. The data were then analysed with the GLIMMIX 


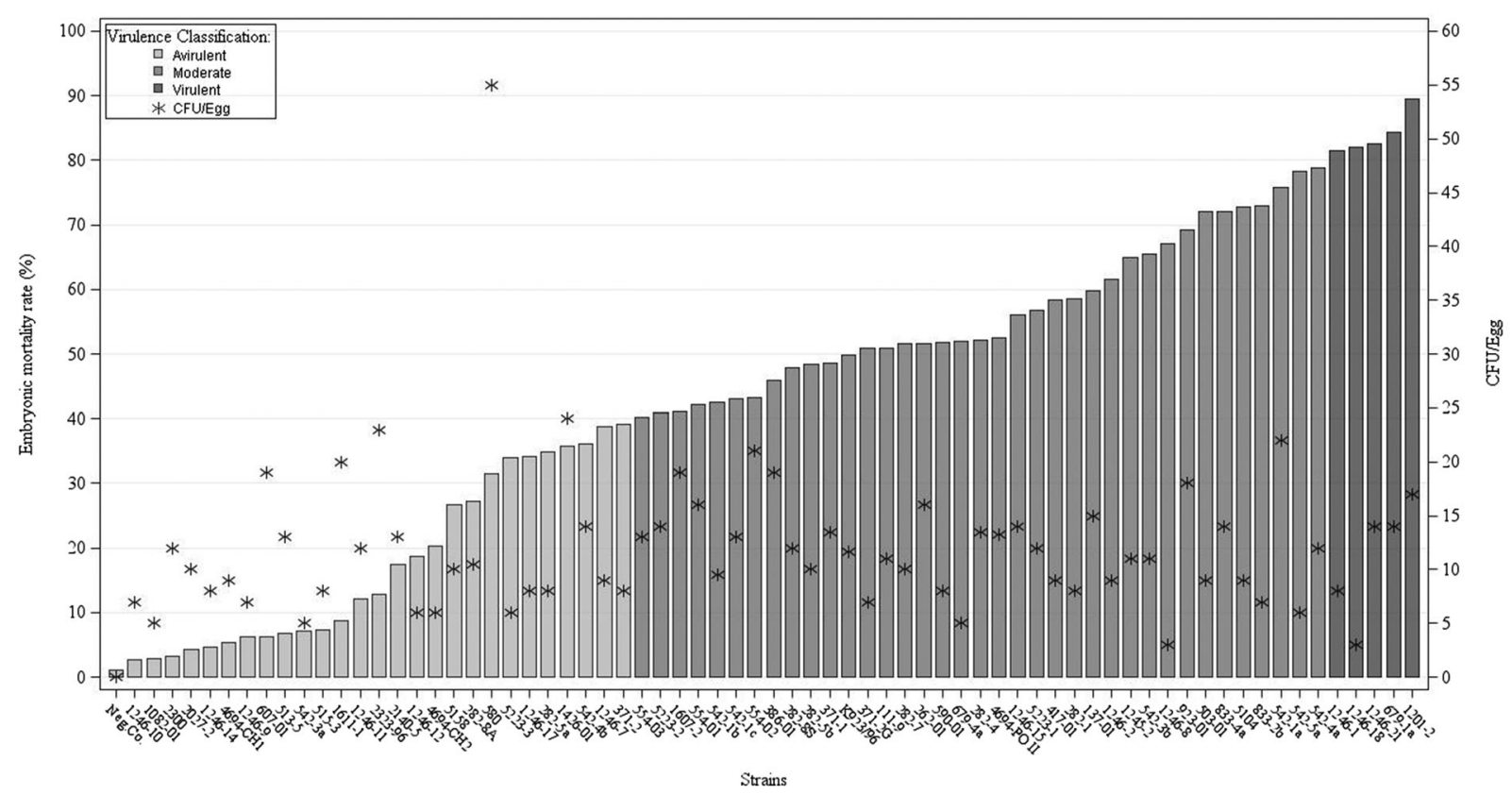

Figure 2. Virulence classification of $68 \mathrm{E}$. faecalis strains, one E. hirae strain (strain 580) and a negative control group (Neg. Co) sorted by the EMR that produce in 10-day-old chicken embryos inoculated into the AC with the represented number of CFU(*). Strains with an EMR below $40 \%(n=27)$ were considered avirulent; from $40 \%$ up to $80 \%$ were classified as strains of moderate virulence $(n=37)$ and above $80 \%$ virulent strains $(n=5)$.

procedure (SAS Institute Inc., 2011) using the following generalized linear model (Littell et al., 1999):

$$
\begin{aligned}
\operatorname{Logit}\left(\pi_{i j}\right) & =\eta_{i j}=\log \left[\pi_{i j} /\left(1-\pi_{i j}\right)\right] \\
& =\phi+b\left(x_{i}\right)+\alpha_{i},
\end{aligned}
$$

where $\eta_{i j}$ denotes the linear predictor, the logit link function is defined by $\log \left[\pi_{i} /\left(1-\pi_{i}\right)\right]=\eta_{i}, \pi_{i j}$ is the probability of mortality until the end of the experiment at 17 days of incubation, $\varphi$ is the overall mean effect, $b$ is the linear regression coefficient of mortality values of the positive control group used in each individual ELA as reference for adjusting the effect of trial $\left(x_{i}\right)$ on embryonic mortality ability and $\alpha_{i}$ is the random effect of the strain.

The mortality produced by each strain was estimated on the logit scale and then back-transformed using the inverse link function $\pi=\exp (x \beta) /[1+\exp (x \beta)]$ to the original scale (probability).

The development of the embryonic survivability over the 7 days p.i. for the different groups of strains classified by its virulence degree quantified by impairment on embryonic mortality ability was illustrated according to the Kaplan-Meier method (survival analysis) by applying the LIFETEST procedure of SAS System 9.3 (SAS Institute Inc., 2011) and using the following model:

$$
\hat{S}(t)=\prod_{j: t_{j} \leq t}\left[1-\frac{d_{j}}{n_{j}}\right] ; \quad \text { for } t_{1} \leq t \leq t_{k}
$$

where $\hat{S}(t)$ is the survivor function and $t$ is the lifetime of a randomly selected experimental unit. For each $j$ : $t_{j} \geq t$, let $t_{1}<t_{2}<\ldots<t_{\mathrm{k}}$ representing the different event times. $n_{j}$ is the number of individuals at risk just prior to $t_{i}$, and $d_{j}$ is the number of individuals that die at time $t_{j}$.

\section{Results}

\section{ERIC-PCR method and phylogenetic analysis}

A phylogenetic tree of one E. hirae and 67 E. faecalis strains based on the aligned nucleotide sequences after ERIC-PCR is shown in Figure 1. A visual comparison of the banding patterns revealed DNA fragments ranging in sizes from 236 to 3380 base pairs. ERIC patterns consisted of four to nine DNA fragments. The phylogenetic analysis of banding pattern showed the most distant relationship to the E. hirae strain, which was implemented as an outgroup. This indicates that the ERIC-PCR can be considered reliable. E. faecalis strains were clustered into five major groups, and only one strain (E. faecalis 1246-15) was unclustered. All clusters showed a remarkable diversity among the $E$. faecalis strains since differences among its banding patterns were observed, except the Cluster 2, whose strains showed identical banding patterns although they were classified into different pathogenicity degrees according to the ELA results. All clusters were composed of avirulent, virulent and moderate virulence strains except five avirulent E. faecalis strains clustered in the Cluster 4 .

\section{Embryo lethality assays}

The ELAs showed different EMRs according to the strain. As shown in Figure 2, the overall EMR of the 
infected embryos ranged from 3\% to $89 \%$ depending on the strain. On the basis of virulence classification of Rudolph (2004), 27 strains, including the E. hirae strain, were classified as avirulent with an EMR below $40 \%$, and five strains were classified as highly virulent with an EMR above $80 \%$. The remaining 37 strains were classified as strains of moderate virulence, whose EMR varied from $40 \%$ to $80 \%$. The average EMR of the negative control group was $1 \%$, and the positive control group (E. faecalis strain K923/96) showed an average EMR of $50 \%$. The E. hirae strain (strain 580) showed low virulence (31\%) although this strain registered the greatest number of bacterial count (55 CFU/egg) during the verification of the CFU number, as shown in Figure 2. The real number of CFU of each strain inoculated into the embryos did not show any clear relationship with the EMR produced. As can be seen in Figure 2, some of the strains with the lowest CFU number were able to produce high embryonic mortality whereas some strains with higher CFU numbers produced low EMRs. For example, $3 \mathrm{CFU} /$ egg of the E. faecalis strains $1246-8$ and 124618 produced an EMR of $78 \%$ and $82 \%$, while 20 and $23 \mathrm{CFU} / \mathrm{ml}$ of the E. faecalis strains $1611-1$ and 232396 produced an EMR of $14 \%$ and $18.4 \%$, respectively.

The lack of relationship between the CFU administered to the embryos and the embryonic mortality produced by each strain was also observed in the positive control group used in all ELAs, as shown in Figure 3. The number of bacteria inoculated into the embryos of the positive control group varied from 4 to $19 \mathrm{CFU} /$ egg among each individual ELAs and did not shown any relationship with the produced embryonic mortality, which ranged from $24 \%$ to $65 \%$.

\section{Embryo survival}

The survival curve for the embryos infected with three groups of strains classified by their virulence degree is presented in Figure 4. Unsurprisingly, embryos infected with avirulent strains had more survival probability than the embryos infected with more virulent strains. However, regardless of the virulence degree of the strains, the highest embryonic mortality occurred at 3 and 4 days p.i., and after 4 days p.i. the embryonic mortality declined substantially.

\section{Macroscopic lesions}

No lesions were observed in the embryos of the negative control group, which received $0.2 \mathrm{ml}$ PBS. However, the infected embryos succumbed to the bacterial infection. The most frequent detrimental effects of artificial infection on embryonic organs and tissues were malformations, growth failure, cranial and skin haemorrhages, subcutaneous oedema and loss of plumage. The detrimental effects resulting from E. faecalis infection on the chicken embryos showed the same pattern regardless of the degree of virulence of the strains. In addition, most severe lesions were observed at 3 and 4 days p.i., and less serious lesions were observed from the fourth day p.i. onwards. Therefore, the most severe lesions were observed when the higher EMR was registered. It should be noted that

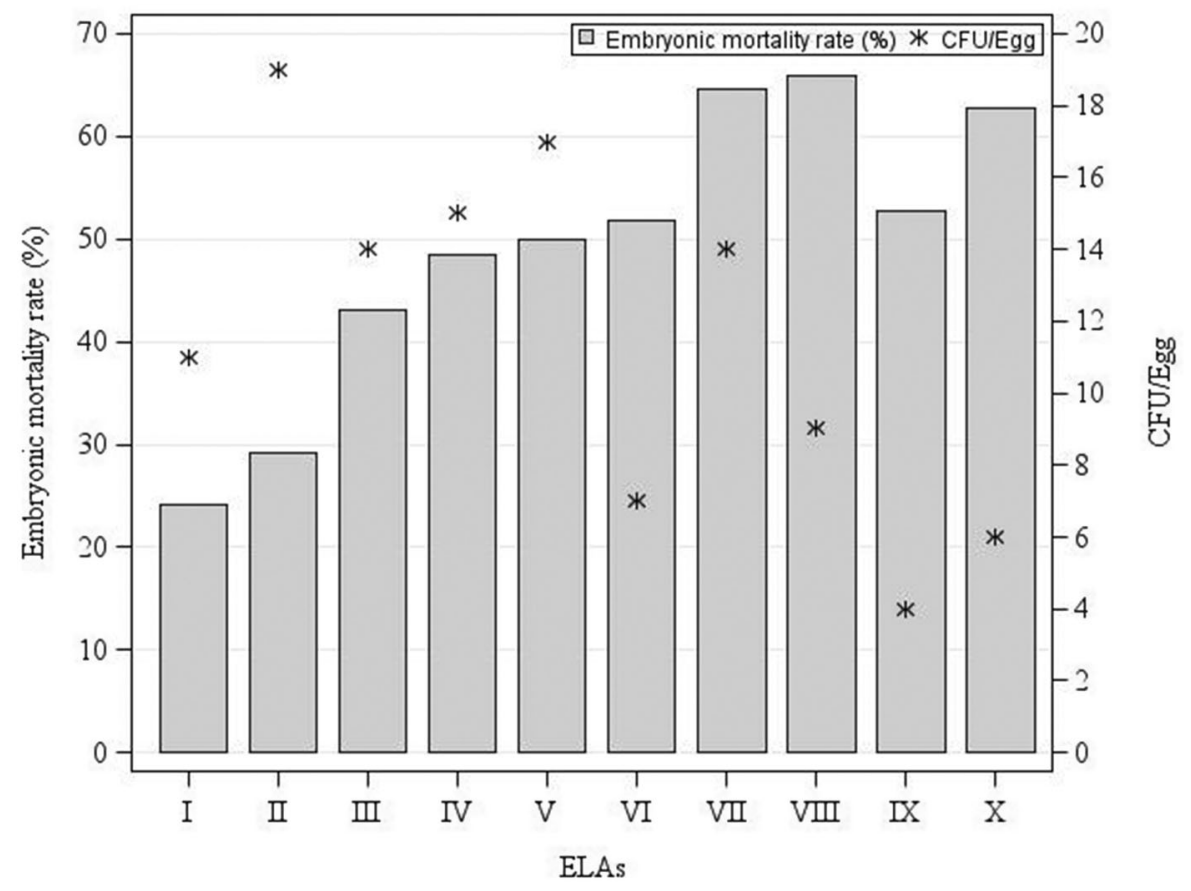

Figure 3. Chicken EMR produced by the E. faecalis strain K923/96 used as positive control in 10 individual ELAs when 10-day-old embryos were inoculated into the AC with the represented number of CFU $\left(^{*}\right)$. 


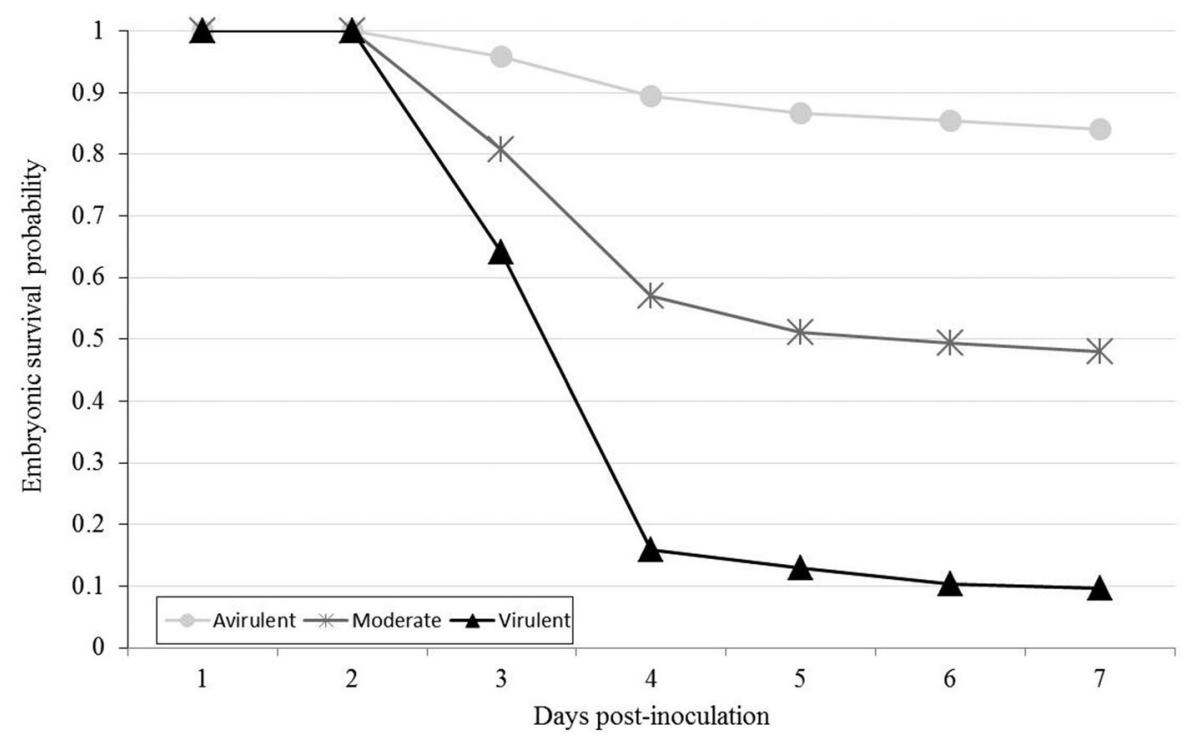

Figure 4. Survival curves of 10-day-old chicken embryos inoculated into the AC with $5 \mathrm{CFU} / \mathrm{ml}$ of three groups of different Enterococcus spp. strains classified by their virulence.

some dead and surviving infected embryos at the end of the ELAs did not show any visible signs.

\section{Re-isolation}

Smear samples from the allantoic fluid of randomly analysed dead embryos as well as from surviving embryos were carried out in order to re-isolate the E. faecalis strains. The bacterial counts of the infected embryos were always positive and their allantoic fluid contained more $\mathrm{CFU} / \mathrm{ml}$ than the infectious dose administered, although some of them did not show any visible lesions or abnormalities (data not shown). The bacterial counts of the negative control group were consistently negative, confirming that there was no cross-contamination.

\section{Discussion}

ERIC-PCR was carried out in order to characterize the genetic diversity of the avian E. faecalis strains and under the hypothesis that strains with similar banding patterns might also show similar infective ability.

ERIC-PCR has been successfully used in previous studies for genotyping of different bacteria such as E. coli (Leung et al., 2004), Vibrio parahaemolyticus (Zulkifli et al., 2009) and L. monocytogenes (Moreno et al., 2013). In the case of Enterococcus spp., Jurkovič et al. (2007) reported that the ERIC-PCR has a high discriminatory power among E. faecium strains isolated from Bryndza cheese in comparison with pulsed-field gel electrophoresis and (GTG) ${ }_{5}$-PCR. Besides, Bachtiar et al. (2015) observed a high genetic diversity among E. faecalis strains from saliva and infected root canal samples by using the ERIC-PCR.
As shown in Figure 1, ERIC-PCR in the present study was able to distinguish Enterococcus spp., specifically E. faecalis and E. hirae strains, which is important to ensure the specificity of the test for E. faecalis. ERIC-PCR revealed a considerable genetic diversity among avian $E$. faecalis strains, which was previously demonstrated in Enterococcus spp. (Jurkovič et al., 2007; Bachtiar et al., 2015). However, since E. faecalis strains with similar banding patterns did not produce the same EMR through the ELA, ERICPCR results did not show a clear relationship to the infection ability of the strains. Similar results were reported by Borst et al. (2014), who observed that an E. cecorium strain with virulent genotype by using the pulsed-field gel electrophoresis did not have a virulent phenotype through the ELA, suggesting that the ELA may be useful in distinguishing strains with the same genotype but different phenotype. Therefore, the molecular taxonomy of Enterococcus spp. might not be able to distinguish between avirulent and virulent strains, as previously reported by Eaton \& Gasson (2001).

The typing of E. faecalis strains has revealed that several STs are related with different human and avian diseases. Specifically, the ST82 of E. faecalis has been related with AA worldwide (Petersen et al., 2007). In this study, from the $49 \mathrm{E}$. faecalis strains that contained the ST82 through the RT-PCR, 10\% were classified as virulent strains, $67 \%$ as moderate virulence and $22 \%$ as avirulent strains through the ELA, as shown in Figure 1. On the contrary, 78\% from the $18 \mathrm{E}$. faecalis strains with absence of the ST82 were classified as avirulent strains and $22 \%$ as strains of moderate virulence. The fact that the E. faecalis strain 1082-1, which is not related to the ST82, was isolated from a field case of AA can be 
explained by the ST172 reported by Petersen et al. (2009), which has been also associated with avian amyloidosis (http://www.mlst.net).

The ELA in the present study has demonstrated its ability to differentiate $E$. faecalis strains with respect to their virulence (Figure 2), in accordance with the ELA results of several authors with different bacterial strains (Wooley et al., 2000; Gibbs et al., 2003; Gibbs \& Wooley, 2003; Rudolph, 2004; Montgomery et al., 2005; Townsend et al., 2008; Stewart-Tull et al., 2009; Jacobsen et al., 2010; Oh et al., 2012; Polakowska et al., 2012; Seo et al., 2013; Borst et al., 2014; Blanco et al., 2017).

The average EMR of the negative control group was $1 \%$, which confirms the appropriate environment in the incubator during the ELAs. Therefore, the obtained embryonic mortalities with each strain can be unequivocally associated to its virulence. The average EMR of the embryos inoculated with $5 \mathrm{CFU} / \mathrm{ml}$ of the E. faecalis strain K923/96 used as positive control in each ELA was $50 \%$, which is in accordance with the results obtained by Blanco et al. (2017), who reported an EMR of $45 \%$ with the same strain and infectious dose. Therefore, although the E. faecalis strain K923/ 96 showed variability between the individual ELAs, as shown in Figure 3, its relative virulence provides accurate results, which is in accordance with the results reported by Gibbs et al. (2003) and Montgomery et al. (2005) with E. coli strains. Hence, these authors suggest the need to be careful when determining the absolute virulence of a strain based on a single ELA.

The EMR which was produced by each strain cannot be explained by the number of CFU administered to the embryos, as shown in Figure 2. This observation is consistent with the results obtained by Gibbs et al. (2003) with E. coli strains, who reported that the ability of a strain to colonize the embryo was not related to its capacity to invade the embryo and cause disease or death. This explains the fact that the number of $\mathrm{CFU} /$ egg of the E. faecalis strain K923/96 used as positive control did not show a clear relationship with the EMR in each individual ELA (Figure 3). However, Seo et al. (2013) reported that virulent and avirulent $R$. anatipestifer strains showed different capacity to replicate in ovo after the inoculation. They observed that the maximum number of $\mathrm{CFU} / \mathrm{ml}$ of a virulent strain was about 1000 times higher than those of an avirulent strain for four days, suggesting that the ability of replication of the strains into embryonic tissues is related to systemic infection of the embryo and death. The E. hirae strain 580, which was inoculated into the embryos with the same infectious dose as the E. faecalis strains, showed the greatest bacterial count administered to the embryos (55 CFU/egg); however, it produced low embryonic mortality (31\%). Therefore, the number of CFU able to produce embryonic mortality seems to depend not only on the strain but also on the species. More research with different species of Enterococcus is required in order to corroborate this theory.

In accordance with the results reported by Wooley et al. (2000), Gibbs et al., (2003), Gibbs \& Wooley (2003), Stewart-Tull et al. (2009), Seo et al. (2013), Borst et al. (2014) and Blanco et al. (2017), regardless of the strain virulence, the survival rate of the embryos decreased faster from 2 to 4 days p.i., whereas the survival rate decreased slower afterwards, as shown in Figure 4. Therefore, the ELA with E. faecalis strains might be optimized by reducing the time of study until 4 days p.i. as Wooley et al. (2000) and Montgomery et al. (2005) have previously reported with E. coli strains. Additionally, Wooley et al. (2000) reported that the survival rate and lesions of the embryos infected with E. coli strains showed a relationship with the degree of strain virulence. Our results also showed a relationship between the embryonic survival rate and the strain virulence since more virulent strains produced a faster decrease in embryonic survival rate from two to four days p.i. than avirulent strains (Figure 4). However, all infected embryos showed the same lesions regardless of the virulence of the strains, in contrast with the results of Wooley et al. (2000) and Borst et al. (2014). The observed macroscopic lesions were similar to those in the broiler embryos infected with E. cecorum by Borst et al. (2014).

No lesions and no re-isolations were observed in the embryos of the negative control group, thus indicating that the embryonic mortality was due to the infection and not due to external effects and, therefore, the research was reliable. On the contrary, the recovered bacterial counts from the allantoic fluid of all analysed infected embryos with E. faecalis strains were always positive, although some of these embryos did not show any visible signs. Besides, an increase in the viable count of the allantoic fluid of dead embryos was observed compared to the initial number of $\mathrm{CFU} / \mathrm{ml}$ contained in the infectious dose. This ability of the bacteria to replicate and invade the embryos after the inoculation, even without killing them, has been previously reported by Wooley et al. (2000), Rudolph (2004), Montgomery et al. (2005), Townsend et al. (2008), Stewart-Tull et al. (2009), Seo et al. (2013) and Blanco et al. (2017).

In conclusion, the results obtained in this study suggest that the ELA can be considered a reliable and useful tool to evaluate the virulence of avian E. faecalis strains by using the EMR. Although the clustering based on the ERIC-PCR did not show a clear relationship with the virulence assessment obtained by the ELA in this study, it can be applied successfully and be useful to obtain the genetic distribution and epidemiology of these bacteria. The results obtained in the present study can be used as a basis for future researches on the E. faecalis virulence, and the high 
pathogenic isolates tested in the present study might be good candidates for autogenous vaccines.

\section{Acknowledgements}

We gratefully acknowledge the technical assistance of the laboratory staff of Lohmann Tierzucht GmbH (Cuxhaven, Germany). We also wish to thank the editor and the anonymous reviewers for positive inputs and suggestions.

\section{Disclosure statement}

No potential conflict of interest was reported by the authors.

\section{ORCID}

Ana E. Blanco (D) http://orcid.org/0000-0001-8949-5035

\section{References}

Andersson, C., Gripenland, J. \& Johansson, J. (2015). Using the chicken embryo to assess virulence of Listeria monocytogenes and to model other microbial infections. Nature Protocols, 10, 1155-1164.

Bachtiar, E.W., Bachtiar, B.M., Dewiyani, S. \& Surono Akbar, S.M. (2015). Enterococcus faecalis with capsule polysaccharides type 2 and biofilm-forming capacity in Indonesians requiring endodontic treatment. Journal of Investigative and Clinical Dentistry, 6, 197-205.

Blanco, A.E., Barz, M., Cavero, D., Icken, W., Sharifi, A.R., Voss, M., Preisinger, R. \& Buxadé, C. (2017). Chicken embryo lethality assay for determining the lethal dose and virulence of Enterococcus faecalis. Avian Pathology, 46, 548-555.

Blanco, A.E., Barz, M., Icken, W., Cavero, D., Mazaheri, A., Voss, M., Schmutz, M. \& Preisinger, R. (2016). Twenty years of amyloid arthropathy research in chickens. World's Poultry Science Journal, 72, 495-508.

Borst, L.B., Suyemoto, M.M., Keelara, S., Dunningan, S.E., Guy, J.S. \& Barnes, H.J. (2014). A chicken embryo lethality assay for pathogenic Enterococcus cecorum. Avian Diseases, 58, 244-248.

Ciftci, A. \& Diker, K.S. (2009). The role of enterococcal virulence factors on experimental amyloid arthropathy in chickens. Kafkas Üniversitesi Veteriner Fakültesi Dergisi, 15, 903-908.

Coburn, P.S., Baghdayan, A.S., Dolan, G.T. \& Shankar, N. (2007). Horizontal transfer of virulence genes encoded on the Enterococcus faecalis pathogenicity island. Molecular Microbiology, 63, 530-544.

Eaton, T.J. \& Gasson, M.J. (2001). Molecular screening of enterococcus virulence determinants and potential for genetic exchange between food and medical isolates. Applied and Environmental Microbiology, 67, 1628-1635.

Felsenstein, J. (2005). PHYLIP (Phylogeny Inference Package) Version 3.695 Distributed by the author. Department of Genome Sciences, University of Washington. Seattle.

Gibbs, P.S., Maurer, J.J., Nolan, L.K. \& Wooley, R.E. (2003). Prediction of chicken embryo lethality with the avian Escherichia coli traits complement resistance, colicin V production, and presence of the increased serum survival gene cluster (iss). Avian Diseases, 47, 370-379.
Gibbs, P.S. \& Wooley, R.E. (2003). Comparison of the intravenous chicken challenge method with the embryo lethality assay for studies in avian colibacillosis. Avian Diseases, 47, 672-680.

Gregersen, R.H., Petersen, A., Christensen, H. \& Bisgaard, M. (2010). Multilocus sequence typing of Enterococcus faecalis isolates demonstrating different lesion types in broiler breeders. Avian Pathology, 39, 435-440.

Han, M.V. \& Zmasek, C.M. (2009). PhyloXML: XML for evolutionary biology and comparative genomics. $B M C$ Bioinformatics, 10, 356.

Hayes, J.R., English, L.L., Carter, P.J., Proescholdt, T., Lee, K.Y., Wagner, D.D. \& White, D.G. (2003). Prevalence and antimicrobial resistance of Enterococcus species isolated from retail meats. Applied and Environmental Microbiology, 69, 7153-7160.

Jacobsen, I.D., Große, K., Slesiona, S., Hube, B., Berndt, A. \& Brock, M. (2010). Embryonated eggs as an alternative infection model to investigate Aspergillus fumigatus virulence. Infection and Immunity, 78, 2995-3006.

Jurkovič, D., Križková, L., Sojka, M., Takáčová, M., Dušinský, R., Krajčovič, J., Vandamme, P. \& Vancanneyt, M. (2007). Genetic diversity of Enterococcus faecium isolated from Bryndza cheese. International Journal of Food Microbiology, 116, 82-87.

Landman, W.J.M., Gruys, E. \& Gielkens, A.L.J. (1998). Avian amyloidosis. Avian Pathology, 27, 437-449.

Landman, W.J.M., Peperkamp, N.H.M.T., Koch, C.A.M., Tooten, P.C.J., Crauwels, P.A.P. \& Gruys, E. (1997). Induction of amyloid arthropathy in chickens. Amyloid, 4, 87-97.

Leung, K.T., Mackereth, R., Tien, Y. \& Topp, E. (2004). A comparison of AFLP and ERIC-PCR analyses for discriminating Escherichia coli from cattle, pig and human sources. FEMS Microbiology Ecology, 47, 111-119.

Littell, R., Milliken, G., Stroup, W. \& Wolfinger, R. (1999). SAS system for mixed models. Raleigh, NC: SAS Institute.

McBride, S.M., Fischetti, V.A., LeBlanc, D.J., Moellering Jr, R.C. \& Gilmore, M.S. (2007). Genetic diversity among Enterococcus faecalis. PLoS ONE, 2, e582.

Montgomery, R.D., Jones, L.S., Boyle, C.R., Luo, Y. \& Boyle, J.A. (2005). The embryo lethality of Escherichia coli isolates and its relationship to various in vitro attributes. Avian Diseases, 49, 63-69.

Moreno, A.M., Paixão, R., Moreno, L.Z., de Gobbi, D.D.S., Raimundo, D.C., Ferreira, T.S.P., Hofer, E., Matte, M.H. \& Moreno, M. (2013). Molecular epidemiology of Listeria monocytogenes isolated from different sources in Brazil. Brazilian Journal of Veterinary Research and Animal Science, 50, 136-144.

Multi Locus Sequence Typing Website (http://www.mlst.net) at Imperial College, London, United Kingdom, developed by Man-Suen Chan and David Aanensen and funded by the Wellcome Trust.

Nolan, L.K., Wooley, R.E., Brown, J., Spears, K.R., Dickerson, H.W. \& Dekich, M. (1992). Comparison of a complement resistance test, a chicken embryo lethality test, and the chicken lethality test for determining virulence of avian Escherichia coli. Avian Diseases, 36, 395-397.

Odinot, P.T., Meis, J.F.G.M., Van Den Hurk, P.J.J.C., Hoogkamp-Korstanje, J.A.A. \& Melchers, W.J.G. (1995). PCR-based characterization of Yersinia enterocolitica: Comparison with biotyping and serotyping. Epidemiology and Infection, 115, 269-277.

Oh, J.Y., Kang, M.S., Yoon, H., Choi, H.W., An, B.K., Shin, E.G. Kim, Y.J., Kim, M.J., Kwon, J.H. \& Kwon, Y.K. (2012). The embryo lethality of Escherichia coli isolates 
and its relationship to the presence of virulence-associated genes. Poultry Science, 91, 370-375.

Olsen, R.H., Frantzen, C., Christensen, H. \& Bisgaard, M. (2012a). An investigation on first-week mortality in layers. Avian Diseases, 56, 51-57.

Olsen, R.H., Schønheyder, H.C., Christensen, H. \& Bisgaard, M. (2012b). Enterococcus faecalis of human and poultry origin share virulence genes supporting the zoonotic potential of E. faecalis. Zoonoses and Public Health, 59, 256-263.

Petersen, A., Bisgaard, M. \& Christensen, H. (2010). Realtime PCR detection of Enterococcus faecalis associated with amyloid arthropathy. Letters in Applied Microbiology, 51, 61-64.

Petersen, A., Christensen, H. \& Bisgaard, M. (2007). Investigations on the existence of a global clone of Enterococcus faecalis associated with amyloid arthropathy in chickens. Proceedings of the Congress of the World Veterinary Poultry Association (p. 254). Beijing, China.

Petersen, A., Christensen, H., Philipp, H.C. \& Bisgaard, M. (2009). Clonality of Enterococcus faecalis associated with amyloid arthropathy in chickens evaluated by multilocus sequence typing (MLST). Veterinary Microbiology, 134, 392-395.

Polakowska, K., Lis, M.W., Helbin, W.M., Dubin, G., Dubin, A., Niedziolka, J.W., Miedzobrodzki, J. \& Wladyka, B. (2012). The virulence of Staphylococcus aureus correlates with strain genotype in a chicken embryo model but not a nematode model. Microbes and Infection, 14, 1352-1362.

Rudolph, B. (2004). Variations Investigations to Enterococcus faecalis as possible factor for etiology of amyloid arthropathy of brown layers. PhD Thesis. Freie Universität Berlin, Germany.

Ruiz-Garbajosa, P., Bonten, M.J., Robinson, D.A., Top, J., Nallapareddy, S.R., Torres, C., Coque, T.M., Canton, R.,
Baquero, F., Murray, B.E. \& del Campo, R. (2006). Multilocus sequence typing scheme for Enterococcus faecalis reveals hospital-adapted genetic complexes in a background of high rates of recombination. Journal of Clinical Microbiology, 44, 2220-2228.

SAS Institute Inc. (2011). SAS/STAT 9.3 user's guide. Cary, NC: Author.

Seo, H.S., Cha, S.Y., Kang, M. \& Jang, H.K. (2013). Chicken embryo lethality assay for determining the virulence of Riemerella anatipestifer isolates. Avian Pathology, 42, 387-392.

Stewart-Tull, D.E.S., Coote, J.G., Thompson, D.H., Candlish, D., Wardlaw, A.C. \& Candlish, A. (2009). Virulence spectra of typed strains of Campylobacter jejuni from different sources: a blinded in vivo study. Journal of Medical Microbiology, 58, 546-553.

Townsend, M.K., Carr, N.J., Iyer, J.G., Horne, S.M., Gibbs, P.S. \& Prüß, B.M. (2008). Pleiotropic phenotypes of a Yersinia enterocolitica flhD mutant include reduced lethality in a chicken embryo model. BMC Microbiology, $8,1-12$.

Wooley, R., Gibbs, P., Brown, T. \& Maurer, J. (2000). Chicken embryo lethality assay for determing the virulence status of avian Escherischia coli isolates. Avian Diseases, 44, 318-324.

World Health Organization. (2012). The evolving threat of antimicrobial resistance: Options for action. Geneva, Switzerland.

Zulkifli, Y., Alitheen, N.B., Son, R., Raha, A.R., Samuel, L., Yeap, S.K. \& Nishibuchi, M. (2009). Random amplified polymorphic DNA-PCR and ERIC PCR analysis on Vibrio parahaemolyticus isolated from cockles in Padang, Indonesia. International Food Research Journal, $16,141-150$. 